\title{
Percolation Transitions and Wetting Transitions in Stochastic Models
}

\author{
Makoto Katori \\ Department of Physics, Faculty of Science and Engineering, \\ Chuo University, Kasuga, Bunkyo-ku, 112-8551 Tokyo, Japan \\ katori@phys.chuo-u.ac.jp
}

Received 1 November 1999

\begin{abstract}
Stochastic models with irreversible elementary processes are introduced, and their macroscopic behaviors in the infinite-time and infinite-volume limits are studied extensively, in order to discuss nonequilibrium stationary states and phase transitions. The Domany-Kinzel model is a typical example of such an irreversible particle system. We first review this model, and explain that in a certain parameter region, the nonequilibrium phase transitions it exhibits can be identified with directed percolation transitions on the spatio-temporal plane. We then introduce an interacting particle system with particle conservation called friendly walkers (FW). It is shown that the $m=0$ limit of the correlation function of $m$ friendly walkers gives the correlation function of the DomanyKinzel model, if we choose the parameters appropriately. We show that FW can be considered as a model of interfacial wetting transitions, and that the phase transitions and critical phenomena of FW can be studied using Fisher's theory of phase transitions in linear systems. The FW model may be the key to constructing a unified theory of directed percolation transitions and wetting transitions. Descriptions of FW as a model of interacting vicious walkers and as a vertex model are also given.
\end{abstract}

\section{Introduction}

Understanding irreversibility in macroscopic systems, based on reversible processes of elementary particles, is one of the motivations of statistical mechanics. In the usual equilibrium statistical mechanics, however, we consider statistically stationary states (invariant measures) in which detailed balance between each elementary process and the corresponding time-reversed process is satisfied. One way to drive the system out of equilibrium is to impose an external force or field and observe the response of the system, or the relaxation back to the equilibrium state after turning off the external force. Another way to study irreversibility is through coarse-grained modeling: We start at a mesoscopic level with elementary processes which are themselves irreversible, and construct a macroscopic system from them [1].

A typical example of models with irreversible elementary processes is the contact process, which is a model of the spread of infection of a disease. In this model system, the elementary processes are (i) spread of a disease from an infected individual to one of its healthy neighbors and (ii) recovery from infection. These processes are irreversible and detailed bal- ance does not hold at all. When an infection rate is low, the disease will be exterminated, while when it is high, coexistence of healthy individuals and infected ones can be observed if the system is large enough. Such a change of state becomes a sharp transition in the double limits of infinite-time and infinite-volume (thermodynamic limit), and defines a nonequilibrium phase transition at a certain critical value of the infection rate. Taking the infinite-time and infinite-volume limits is necessary to have a mathematically well defined macroscopic system constructed from mesoscopic levels. The supercritical phase in which healthy and infected individuals coexist is a typical example of a nonequilibrium stationary state. Detailed descriptions of such nonequilibrium systems will be one of the starting points to construct a general theory of nonequilibrium statistical mechanics.

In the present paper, we consider the discretetime version of contact processes, which is called the Domany-Kinzel model [2] and a related process called friendly walkers (FW) [3], and discuss two kinds of phase transitions, directed percolation (DP) transitions and interfacial wetting transitions. In Section II we define the Domany-Kinzel model and give its percolation representation on the spatio-temporal plane in a cer- 
tain parameter region. Using this representation, we can easily understand that nonequilibrium phase transitions in the Domany-Kinzel model (as well as the contact process) can be identified with the DP transitions in two dimensions. Though DP is a famous unsolved model even in two dimensions, rigorous lower and upper bounds of the critical line on a phase diagram have been demonstrated; they are reviewed there. Then we introduce FW with two parameters in Section III. The Domany-Kinzel model involves creation and annihilation of particles, while the number of walkers is conserved in FW. We can prove, however, however, the $m=0$ limit of the correlation function of $m$ friendly walkers gives the correlation function of the DomanyKinzel model, when we impose a certain relation among parameters. In Section IV we show that, if we identify the spatio-temporal plane on which trajectories of FW are described with two-dimensional space, the $m$ FW can be regarded as a model of interfacial wetting transitions in a two-dimensional $(m+1)$-phase systems, where $\mathrm{FW}$ trajectories describe the configurations of interfaces; Fisher's theory of phase transitions in such systems [4] can be applied. The generating functions of two FW can be evaluated exactly by a combinatorial method. Combining this exact result and Fisher's theory, the critical line and critical exponents are determined for $m=2$. Two-dimensional wetting transitions are usually modelled by vicious walkers $[4,5,6]$. Section $\mathrm{V}$ is devoted to the possible description of $\mathrm{FW}$ as interacting vicious walkers and as a vertex model in two dimensions.

At the end of Section III we claim that the DomanyKinzel model can be regarded as a "grand-canonical" ensemble of FW in a sense. This new point of view lets us consider the possibility of applying standard methods of statistical mechanics, in the canonical ensemble, to the $m$-FW problem. Using the exactly solved case, $m=2$, we demonstrate in Section V that the asymptotic form of the partition function of the vertex model, which is equivalent to two $\mathrm{FW}$, can be determined by the Bethe ansatz method. Generalization of the present demonstration for $m \geq 3$ is a challenging open problem.

\section{Directed Percolation (DP) Transitions in Irreversible Stochastic Models}

\section{II.1 The Domany-Kinzel Model}

Domany and Kinzel $[2,7]$ introduced a class of discrete-time processes $\eta_{t}$ on the $1+1$ dimensional spatio-temporal plane

$$
V=\left\{(x, t) \in \mathbf{Z}^{2}: x+t=\text { even, } t=0,1,2, \cdots\right\},
$$

where $\mathbf{Z}=\{\cdots,-2,-1,0,1,2, \cdots\}$. Their models are stochastic cellular automata, which simulate creation and annihilation processes of particles caused by shortranged interactions among them [8]. Each site $x \in V$ exists in one of two states, 0 (vacant) or 1 (occupied by a particle). Let $\mathbf{Z}_{\mathrm{e}}=\{\cdots,-4,-2,0,2,4, \cdots\}$ and $\mathbf{Z}_{\mathrm{o}}=\{\cdots,-3,-1,1,3, \cdots\}$; the state at time $t=$ even (resp. odd) is given by the set $\eta_{t}$ of sites occupied by particles $\eta_{t} \subset \mathbf{Z}_{\mathrm{e}}$ (resp. $\mathbf{Z}_{\mathrm{o}}$ ). The time evolution of state $\eta_{t}$ is given by the following stochastic rule,

$$
\operatorname{Prob}\left(x \in \eta_{t+1} \mid \eta_{t}\right)=f\left(\left|\eta_{t} \cap\{x-1, x+1\}\right|\right),
$$

where $\operatorname{Prob}\left(\omega_{1} \mid \omega_{2}\right)$ is the conditional probability of $\omega_{1}$ given $\omega_{2},|A|$ denotes the number of sites included in a set $A$, and a function $f$ is parameterized by $p_{1}$ and $p_{2}$ as

$$
f(N)= \begin{cases}0 & N=0 \\ p_{1} & N=1 \\ p_{2} & N=2\end{cases}
$$

with $0 \leq p_{1}, p_{2} \leq 1$. Fig. 1(a) shows the elementary processes. We assume that the initial state is a finite set $A \subset \mathbf{Z}_{\mathrm{e}},|A|<\infty$, and write the process starting from $A$ as $\eta_{t}^{A}$. In particular, when $A$ is a singleton $\{y\}, y \in \mathbf{Z}_{\mathrm{e}}$, we simply write $\eta_{t}^{y}$. For example, $\eta_{t}^{0}$ is the process starting from only one particle at the origin. Since $f(0)=0$, the state in which all sites are vacant is an absorbing state and the process is irreversible.

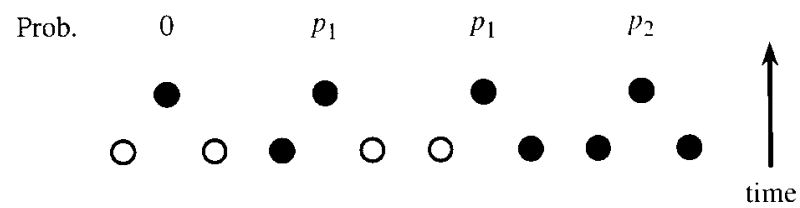

(a)

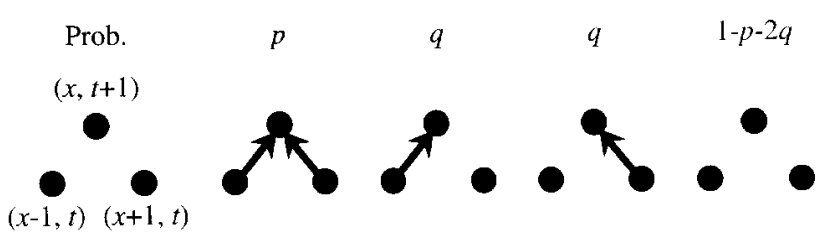

(b)

Figure 1. (a) The elementary processes of the DomanyKinzel model $\eta_{t}$. The full (resp. open) denote sites occupied by particles (resp. empty site). (b) The gadgets for the directed percolation representation.

The Domany-Kinzel model can be regarded as the discrete-time version of the contact process. The contact process is a continuous-time stochastic process on a 
lattice (an interacting particle system) [9-11,1,12]. The state at time $t$ is given by the set $\xi_{t} \in \mathbf{Z}$ of lattice sites which are occupied by particles. The system evolves as follows:

(i) if $x \in \xi_{t}$, then $x$ becomes vacant at rate 1 ,

(ii) if $x \notin \xi_{t}$, then $x$ becomes occupied at rate $g\left(\left|\xi_{t} \cap\{x-1, x+1\}\right|\right)$ with

$$
g(N)= \begin{cases}0 & N=0 \\ \lambda & N=1 \\ \theta \lambda & N=2\end{cases}
$$

where $\lambda$ and $\theta$ are non-negative parameters [13-15]. Fig. 2 illustrates the above elementary processes. The contact process is a model of spread of an infectious disease in one dimension. An individual at $x \in \mathbf{Z}$ is considered infected if $x \in \xi_{t}$, and healthy if $x \notin \xi_{t}$. The parameter $\lambda$ is the infection rate in the case that one of the neighbors is infected. In the case where both neighbors are infected the infection rate is given by $\lambda$ multiplied by $\theta$. The process with $\theta=2$ is usually called the basic contact process, and was first studied by Harris [16]. The process with $\theta=1$ is called the threshold contact process by Liggett [17]. The basic contact process is equivalent to the Reggeon quantum spin model in high-energy physics [18-20]. Dickman and Burschka [21] studied a nonequilibrium lattice model as a simplified version of the model for catalytic surfaces proposed by Ziff et al [22]. Their model is called the A model but is equivalent to the threshold contact process. See also Dickman and Tomé [23] for other related models.
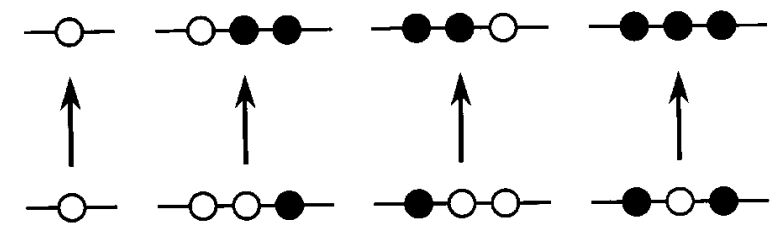

rates 1

$\lambda$

$\lambda$

$\theta \lambda$

Figure 2. The elementary processes of the contact process. The full circles denote particles and the open circles denote vacancies.

The contact process exhibits a nonequilibrium phase transition from the extinction phase to the survival phase upon increasing the infection rate $\lambda$, for each $\theta$. The former phase, in which unique stationary state is the trivial absorbing state, represents the extermination of disease, while in the latter phase the process has a nontrivial, nonequilibrium stationary state, which represents the coexistence of infected and healthy individuals. The phase diagram of the contact process was studied in $[14,15,24]$. The Domany-Kinzel model shows nonequilibrium phase transitions which are similar to the transitions of the contact process.

\section{II.2 DP Transitions}

In the parameter region

$$
p_{1} \leq p_{2} \leq 2 p_{1}
$$

we see direct correspondence between the phase transitions of the Domany-Kinzel model and directed percolation transitions $[2,7,10,25]$. As shown in Fig.1 (b), at each triangle of three sites $(x-1, t),(x+1, t)$ and $(x, t+1)$, the following 'gadgets' are placed independently: Two arrows with probability $p$, one arrow with probability $q$ (to the northeast and to the northwest, respectively), and nothing with probability $1-p-2 q$. The arrow means that the directed bond from the site at the bottom of the arrow to the site at the top of the arrow is open. If there is a sequence $\left(x_{0}, t\right)=$ $(x, t),\left(x_{1}, t+1\right), \cdots,\left(x_{n}, t+n\right)=(y, t+n)(n \geq 1)$ of sites in $V$ such that for each $0 \leq i \leq n-1$ the bond from $\left(x_{i}, t+i\right)$ to $\left(x_{i+1}, t+i+1\right)$ is open, this sequence is called an open path from $(x, t)$ to $(y, t+n)$ and we write $(x, t) \longrightarrow(y, t+n)$. Then for a set $A \subset \mathbf{Z}_{\mathrm{e}}$ we define a set $\hat{\eta}_{t}^{A}$ by

$$
\hat{\eta}_{t}^{A}=\bigcup_{x: x \in A}\{y:(x, 0) \longrightarrow(y, t)\} .
$$

It is easy to confirm that if and only if

$$
p_{1}=p+q \quad \text { and } \quad p_{2}=p+2 q
$$

the process $\eta_{t}^{A}$ that evolves by (2) with (3) from the initial state $A \subset \mathbf{Z}_{\mathrm{e}}$ is equal to $\hat{\eta}_{t}^{A}$. Since we must have $0 \leq p, q \leq 1$ and $0 \leq 1-p-2 q \leq 1$, this directed percolation representation of the process is possible if and only if (5) is satisfied.

In the parameter region (5), the Domany-Kinzel model includes the following percolation models as special cases.

directed site percolation (DSP) with site concentration $\alpha$

$$
p_{1}=p_{2}=\alpha \quad(p=\alpha, q=0)
$$

directed bond percolation (DBP) with bond concentration $\beta$

$$
p_{1}=\beta, p_{2}=\beta(2-\beta) \quad\left(p=\beta^{2}, q=\beta(1-\beta)\right)
$$

directed site-bond percolation with site (resp. bond) concentration $\alpha$ (resp. $\beta$ ) 
$p_{1}=\alpha \beta, p_{2}=\alpha \beta(2-\beta) \quad\left(p=\alpha \beta^{2}, q=\alpha \beta(1-\beta)\right)$

When (5) is satisfied, we can define a percolation transition for the Domany-Kinzel model. Consider the probability

$$
\theta_{t}\left(p_{1}, p_{2}\right)=\operatorname{Prob}\left(\eta_{t}^{0} \neq \emptyset\right)
$$

In the parameter region (5), since the percolation representation ensures that it is non-increasing in $t$, the infinite-time limit is well-defined,

$$
\theta\left(p_{1}, p_{2}\right)=\lim _{t \rightarrow \infty} \theta_{t}\left(p_{1}, p_{2}\right) .
$$

(It is easy to prove that $\theta\left(p_{1}, p_{2}\right)=0$ for $0 \leq p_{1} \leq 1 / 2$, $0 \leq p_{2} \leq 1$ [10]. ) For each $0 \leq p_{2} \leq 1, \theta\left(p_{1}, p_{2}\right)$ is a non-decreasing function of $p_{1}$, if $p_{1} \leq p_{2}$, and we can uniquely define the critical value $p_{1 \mathrm{c}}\left(p_{2}\right)$ by using $\theta\left(p_{1}, p_{2}\right)$ as an order parameter of the percolation transition as

$$
\begin{aligned}
p_{1 \mathrm{c}}\left(p_{2}\right) & =\sup \left\{0 \leq p_{1} \leq 1: \theta\left(p_{1}, p_{2}\right)=0\right\} \\
& =\inf \left\{0 \leq p_{1} \leq 1: \theta\left(p_{1}, p_{2}\right)>0\right\}
\end{aligned}
$$

We call $\theta_{t}\left(p_{1}, p_{2}\right)$ the $t$-step percolation probability and $\theta\left(p_{1}, p_{2}\right)$ the percolation probability of the DomanyKinzel model with parameters $p_{1}, p_{2}$. In the region (5), $p_{1}=p_{1 \mathrm{c}}\left(p_{2}\right), 0 \leq p_{2} \leq 1$, gives a critical line on the $\left(p_{1}, p_{2}\right)$-phase diagram. The $p_{1}$ coordinates of the crossing points of this critical line with the lines $p_{2}=p_{1}$ and $p_{2}=p_{1}\left(2-p_{1}\right)$ give the critical concentrations $\alpha_{\mathrm{c}}$ and $\beta_{\mathrm{c}}$ of DSP and DBP, respectively.

Rigorous lower and upper bounds for the critical line (13) were calculated in the region (5) by Katori and Tsukahara [25] and by Liggett [26], respectively. Let

$$
\begin{aligned}
B_{\ell}\left(p_{1}, p_{2}\right)= & \left\{1-p_{1}\left(p_{2}-p_{1}\right)\right\}\left\{1-\left(p_{2}-p_{1}\right)\right\} \\
& \times\left[1-\left\{1-\left(2 p_{1}-p_{2}\right)\right\} p_{1}-\left(p_{2}-p_{1}\right)^{2}\left(1-p_{1}\right)\right] b_{1}\left(p_{1}, p_{2}\right) \\
& +\left\{p_{2}-p_{1}\left(2-p_{1}\right)\right\} p_{1}^{2}\left(p_{2}-1\right)\left(p_{1}-1\right)^{2} b_{2}\left(p_{1}, p_{2}\right)
\end{aligned}
$$

with

$$
\begin{aligned}
b_{1}\left(p_{1}, p_{2}\right)= & 2 p_{1}^{6}-\left(p_{2}+2\right) p_{1}^{5}-\left(p_{2}^{2}-2 p_{2}-2\right) p_{1}^{4}+\left(3 p_{2}^{2}-3 p_{2}-1\right) p_{1}^{3} \\
& -4 p_{2}\left(p_{2}-1\right) p_{1}^{2}+\left(3 p_{2}-1\right)\left(p_{2}-1\right) p_{1}-\left(p_{2}-1\right)^{2}, \\
b_{2}\left(p_{1}, p_{2}\right)= & \left(p_{2}+1\right) p_{1}^{6}-\left(3 p_{2}^{2}+3 p_{2}-1\right) p_{1}^{5}+p_{2}^{2}\left(3 p_{2}+4\right) p_{1}^{4} \\
& -\left(p_{2}^{4}+3 p_{2}^{3}+4 p_{2}^{2}-p_{2}-2\right) p_{1}^{3}+\left(p_{2}^{4}+4 p_{2}^{3}-p_{2}^{2}-2 p_{2}+1\right) p_{1}^{2} \\
& -\left(p_{2}-1\right)\left(p_{2}^{3}+p_{2}^{2}+2 p_{2}+1\right) p_{1}+\left(p_{2}+1\right)\left(p_{2}-1\right)^{2},
\end{aligned}
$$

and

$$
B_{\mathrm{u}}\left(p_{1}, p_{2}\right)=p_{2}-4 p_{1}\left(1-p_{1}\right) .
$$

Define

$$
\begin{aligned}
& p_{1 \ell}\left(p_{2}\right)=\sup \left\{p_{1}: p_{2} / 2 \leq p_{1} \leq p_{2} \text { and } B_{\ell}\left(p_{1}, p_{2}\right)<0\right\} \\
& p_{1 \mathrm{u}}\left(p_{2}\right)=\sup \left\{p_{1}: p_{2} / 2 \leq p_{1} \leq p_{2} \text { and } B_{\mathrm{u}}\left(p_{1}, p_{2}\right)>0\right\}
\end{aligned}
$$

Then $[25,26]$

$$
p_{1 \ell}\left(p_{2}\right) \leq p_{1 \mathrm{c}}\left(p_{2}\right) \leq p_{1 \mathrm{u}}\left(p_{2}\right) .
$$

For the DSP (8) and the DBP (9) cases, (19) gives

$$
0.688 \leq \alpha_{\mathrm{c}} \leq 3 / 4, \quad 0.626 \leq \beta_{\mathrm{c}} \leq 2 / 3,
$$

where the lower bound of $\beta_{\mathrm{c}}$ was originally given by
Dhar [27]. The most reliable values for $\alpha_{\mathrm{c}}$ and $\beta_{\mathrm{c}}$ estimated so far are given by Jensen and Guttmann [28] by the series expansion method as $\alpha_{\mathrm{c}}=0.705485 \pm$ 0.000005 and $\beta_{\mathrm{c}}=0.6447006 \pm 0.0000010$.

Fig. 9 in [25] shows the lower and upper bounds in the $\left(p_{1}, p_{2}\right)$-plane. We can see that $\lim _{p_{2} \rightarrow 1} p_{1 \ell}\left(p_{2}\right)=$ 
$\lim _{p_{2} \rightarrow 1} p_{1 \mathrm{u}}\left(p_{2}\right)=1 / 2$ and then (19) determines $p_{1 \mathrm{c}}(1)=1 / 2$. Exact values of $p_{1 \mathrm{c}}\left(p_{2}\right)$ are not known except for this special case $p_{2}=1$. Though we do not know the exact location of the critical line, the bounds (19) ensure that both extinction phase (non-percolation phase) and the survival phase (percolation phase) are non-empty in the phase diagram, and we certainly observe directed percolation transitions in the region (5) of the Domany-Kinzel model.

\section{Friendly Walkers (FW)}

\section{III.1 Definition of FW}

Let us consider an enumeration problem of weighted paths of $m(\geq 1)$ walkers on $\mathbf{Z}$ with discrete time. At time $t=0$, all walkers are at the origin and they move simultaneously in time. At each time step $t \rightarrow t+1$, each walker steps either to the right or left nearestneighbor site with equal probability. Two or more walkers can occupy the same site on $\mathbf{Z}$ and they can walk together. We call a set of walkers which occupy the same site a group. (When there is only one walker at a site, the group is a singleton.) We label the $m$ walkers by integers $1,2, \cdots m$ and the location of the $i$-th walker at time $s$ is written as $x_{i}(s)$. We impose the following non-crossing condition.

Non-Crossing Condition: For any $s \geq 0$,

$$
x_{1}(s) \leq x_{2}(s) \leq \cdots \leq x_{m}(s) .
$$

We introduce two parameters $p \geq 0$ and $\tau \geq 0$; the weights of the movement of the $m$ walkers are determined as follows.

Weight $p^{s_{s}}$ : At each time $1 \leq s \leq t$, let $s_{s}$ be the number of groups (i.e. $s_{s}=$ $\left.\left|\left\{x_{1}(s), x_{2}(s), \cdots, x_{m}(s)\right\}\right|\right)$. Then we include a factor $p^{s_{s}}$ in the weight.

Weight $\tau^{\ell_{s}}$ : At each time $1 \leq s \leq t$, let $\ell_{s}$ be the number of distinct sites at which two different groups of walkers join together at that time, the two groups having been separate at the previous time. We include a factor $\tau^{\ell_{s}}$ in the weight.
When two or more walkers walk together along the same path, $s_{s}$ remains small, but if they tend to walk separately, then $s_{s}$ increases and the weight $p^{s_{s}}$ decreases, since $0 \leq p \leq 1$. This shows that the walkers prefer to work together than walk alone. For this reason, they are called friendly walkers (FW) [3,29,30]. Fig. 3 (a) shows an example of trajectories of the three $(m=3)$ FW up to $t=17$.

\section{III.2 Generating Functions of FW}

It should be noted that the set of sites $(x, t)$ on $\mathbf{Z}^{2}$, on which walkers can pass up to time $t \geq 0$, defines a downward-pointing triangular region in the lattice

$$
V_{t}=\left\{(x, s) \in \mathbf{Z}^{2}: x+s=\text { even, } 0 \leq s \leq t\right\} .
$$

The trajectory of one walker is expressed by a path, which is a sequence of successive bonds, and a set of trajectories of $m$ walkers is a union of such paths.

We consider a union of paths, each of which starts from the origin $(0,0)$ and terminates at one of the sites in $\left\{\left(x_{1}, t\right),\left(x_{2}, t\right), \cdots,\left(x_{k}, t\right)\right\}$, where $x_{1}<x_{2}<$ $\cdots<x_{k}$. Such a union of paths makes a graph $g$ with $\left.g\right|_{0}=\{0\}$ and $\left.g\right|_{t}=\left\{x_{1}, x_{2}, \cdots, x_{k}\right\}$. We define $\Gamma_{t}\left(0 ; x_{1}, x_{2}, \cdots, x_{k}\right)$ to be the set of all such graphs. Each graph $g \in \Gamma_{t}\left(0 ; x_{1}, x_{2}, \cdots, x_{k}\right)$ is characterized by the following quantities,

$$
\begin{aligned}
& s(g)=\text { the number of sites in } g, \\
& b(g)=\text { the number of bonds in } g, \\
& \ell(g)=\text { the number of loops in } g .
\end{aligned}
$$

By definition, $s(g)=\sum_{s=1}^{t} s_{s}$ and $\ell(g)=\sum_{s=2}^{t} \ell_{s}$, where $s_{s}$ and $\ell_{s}$ are powers of weights $p^{s_{s}}$ and $\tau^{\ell_{s}}$ for the $s$-th step of the FW.

For a given graph $g \in \Gamma_{t}\left(0 ; x_{1}, x_{2}, \cdots, x_{k}\right)$, however, there may be many different realizations of $m \mathrm{FW}$, which correspond to the same graph $g$. Let $c(g ; m)$ be the number of the distinct realizations of $m$ FW corresponding to graph $g$. The $(k+1)$-point generating function of $m$ friendly walkers is defined as

$$
Z_{t}\left(p, \tau ; 0 ; x_{1}, x_{2}, \cdots, x_{k} ; m\right)=\sum_{g: g \in \Gamma_{t}\left(0 ; x_{1}, x_{2}, \cdots, x_{k}\right)} c(g ; m) \tau^{\ell(g)} p^{s(g)-1} .
$$

Then we define the generating function of $m \mathrm{FW}$ as

$$
Z_{t}(p, \tau ; m)=\sum_{k: k \geq 1} \sum_{\left\{x_{i}\right\}: x_{1}<x_{2}<\cdots<x_{k}} Z_{t}\left(p, \tau ; 0 ; x_{1}, x_{2}, \cdots, x_{k} ; m\right) .
$$




\section{III.3 Relation between DP and FW}

Define the $(k+1)$-point correlation function

$$
C_{t}\left(p_{1}, p_{2} ; 0 ; x_{1}, x_{2}, \cdots, x_{k}\right)=\operatorname{Prob}\left(\eta_{t}^{0}\left(x_{i}\right)=1 \text { for } 1 \leq i \leq k\right)
$$

for the Domany-Kinzel model, where $\left\{x_{1}, x_{2}, \cdots, x_{k}\right\} \subset \mathbf{Z}_{\mathrm{e}}$ (resp. $\mathbf{Z}_{\mathrm{o}}$ ) for $t=$ even (resp. odd), and we assume that $x_{1}<x_{2}<\cdots<x_{k}$. Arrowsmith and Essam [31] proved the following formula for directed site-bond percolation (10),

$$
C_{t}\left(\alpha \beta, \alpha \beta(2-\beta) ; 0 ; x_{1}, x_{2}, \cdots, x_{k}\right)=\sum_{g: g \in \Gamma_{t}\left(0 ; x_{1}, x_{2}, \cdots, x_{k}\right)}(-1)^{\ell(g)} \alpha^{s(g)-1} \beta^{b(g)},
$$

where $\alpha$ and $\beta$ are the site and bond concentrations, respectively. This is called the low-density expansion formula, since the right-hand side is a power series with respect to the concentrations $\alpha$ and $\beta$; it has been used for calculating series expansions for the pair-connectedness functions for directed percolation models [32, 33]. This formula can be generalized for the Domany-Kinzel model as

$$
C_{t}\left(p_{1}, p_{2} ; 0 ; x_{1}, x_{2}, \cdots, x_{k}\right)=\sum_{g: g \in \Gamma_{t}\left(0 ; x_{1}, x_{2}, \cdots, x_{k}\right)}(-1)^{\ell(g)}\left(\frac{2 p_{1}-p_{2}}{p_{1}}\right)^{\ell(g)} p_{1}^{s(g)-1} .
$$

The proof is given in [34].

So far we have assumed that $m$ is an integer, but we can allow $m$ be any real number, since $c(g ; m)$ is a polynomial in $m$ and the generating functions of the $m \mathrm{FW}$ depend on $m$ only through $c(g ; m)$. Cardy and Colaiori [29] proved

$$
\lim _{m \rightarrow 0} c(g ; m)=(-1)^{k-1+\ell(g)}
$$

for $g \in \Gamma_{t}\left(0 ; x_{1}, x_{2}, \cdots, x_{k}\right)$. Then we have

$$
C_{t}\left(p_{1}, p_{2} ; 0 ; x_{1}, x_{2}, \cdots, x_{k}\right)=\lim _{m \rightarrow 0}(-1)^{k-1} Z_{t}\left(p_{1}, \frac{2 p_{1}-p_{2}}{p_{1}} ; 0 ; x_{1}, x_{2}, \cdots, x_{k} ; m\right) .
$$

Let $\theta_{t}\left(p_{1}, p_{2}\right)$ and $Z_{t}(p, \tau ; m)$ be the $t$-step percolation probability of the Domany-Kinzel model defined by (11) and the generating function of the $m \mathrm{FW}$ defined by (25). By the principle of inclusion and exclusion

$$
\theta_{t}\left(p_{1}, p_{2}\right)=\sum_{k: k \geq 1}(-1)^{k-1} \sum_{\left\{x_{i}\right\}: x_{1}<x_{2}<\cdots<x_{k}} C_{t}\left(p_{1}, p_{2} ; 0 ; x_{1}, x_{2}, \cdots, x_{k}\right)
$$

we can conclude that

$$
\theta_{t}\left(p_{1}, p_{2}\right)=\lim _{m \rightarrow 0} Z_{t}\left(p_{1}, \frac{2 p_{1}-p_{2}}{p_{1}} ; m\right)
$$

By the definition of $c(g ; m)$, if $m$ is fixed as a nonzero finite integer, $c(g ; m)=0$ for graphs having $m+1$ or more sites on a constant-time line (horizontal line) in $V_{t}$. This means that $Z_{t}\left(p, \tau ; 0 ; x_{1}, x_{2}, \cdots, x_{k} ; m\right)$ is a partial sum of the graphs $g$ in $\Gamma_{t}\left(0 ; x_{1}, x_{2}, \cdots, x_{k}\right)$. On the other hand, (29) shows that $c(g ; 0) \neq 0$ for any $g$ and the formula (28) asserts that the total summation of all graphs $g \in \Gamma_{t}\left(0 ; x_{1}, x_{2}, \cdots, x_{k}\right)$ gives the $(k+1)$-point correlation function for the DomanyKinzel model. This is reason why we can obtain the Domany-Kinzel model, in which particles are created and annihilated, as a limit of FW, in which particles are conserved. If we consider the $m \mathrm{FW}$ as a canon-

ical system with a fixed number of particles $m$, then we can say that the Domany-Kinzel model is a "grandcanonical" ensemble of FW.

\section{Wetting Transitions}

\section{IV.1 Fisher's Theory of Phase Transi- tions in Linear Systems}

The relations (30) and (32) imply that the DomanyKinzel model can be considered as the $m=0$ limit of the $m$-FW problem. As explained in Section II, the Domany-Kinzel model exhibits DP-like transitions. What are the corresponding phase transitions in systems of $m \mathrm{FW}$ ? Since the order parameter (12) of the DP transition is defined as a $t \rightarrow \infty$ limit of $\theta_{t}\left(p_{1}, p_{2}\right)$, 
the asymptote of $Z_{t}(p, \tau ; m)$ for $t \gg 1$ should be studied in the $m \mathrm{FW}$.

In Fig.3 (a), which shows an example of a set of trajectories of 3-FW, we can classify the time intervals into two types: The intervals in which all walkers walk together (i.e. there is only one group), and those in which they are separated into two or more groups. If we ignore the details of the ways of walking in the latter intervals, the set of trajectories can be represented by a simple linear system in the form of a "necklace" as shown in Fig.3 (b).

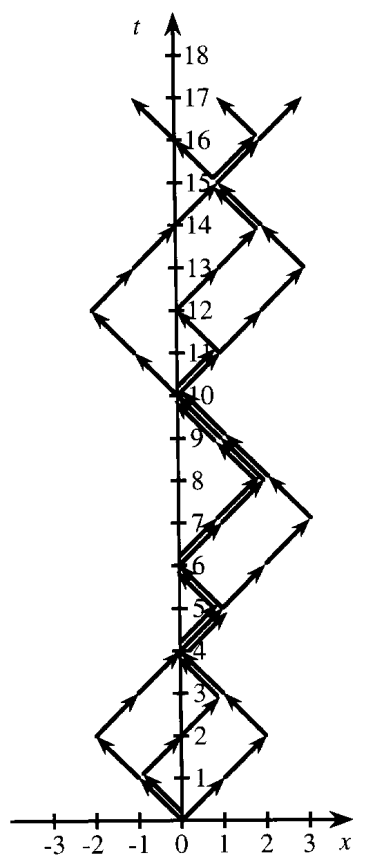

(a)

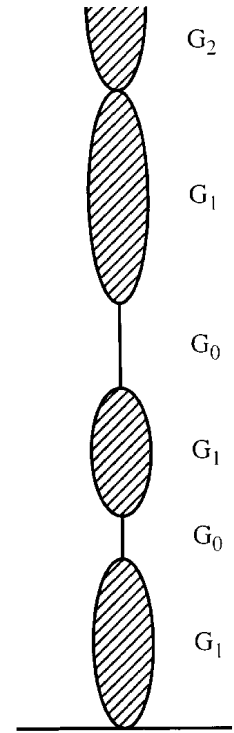

(b)
Figure 3. (a) An example of trajectories of three $(m=3)$ FW up to $t=17$. (b) A linear system in the form of a "necklace".

Fisher discussed a simple but general mathematical mechanism which makes such linear systems to display phase transitions [4]. In his paper, many possible applications of his theory to physical phenomena are given. We select one of them here, an interfacial wetting transition in a two-dimensional $(m+1)$-phase system. Fig. 4 (a) illustrates the system, in which the $B_{1}, \cdots, B_{m-1}$ phases are confined between the bulk $A$ and bulk $C$ phases. As a parameter (e.g., the temperature of the system) is varied, the interface $A \| C$ becomes wet by intermediate phases $B_{1}, \cdots, B_{m-1}$, that is , a wetting transition occurs (Fig.4(b)). Now we borrow Fisher's theory and discuss the phase transitions and critical phenomena of the $m \mathrm{FW}$.

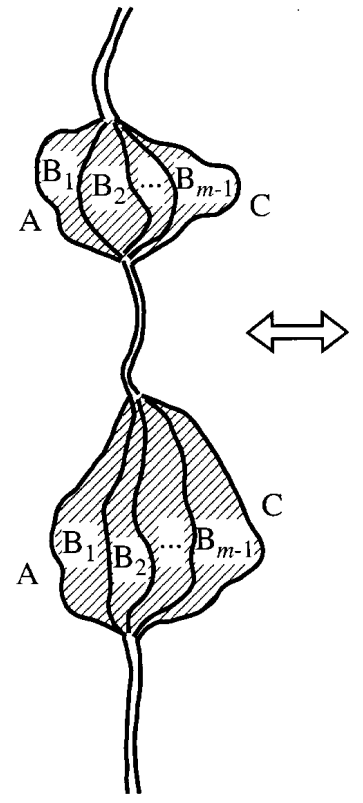

(a)

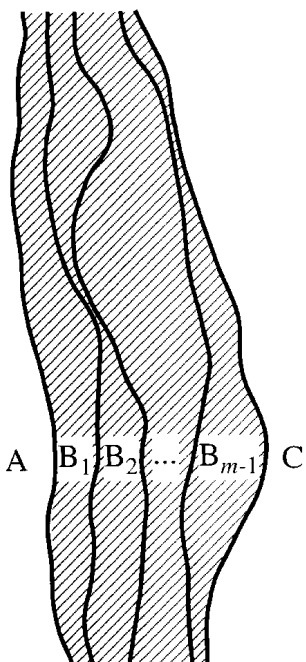

(b)
Figure 4. A interfacial wetting transition from the (a) nonwetting phase to the $(\mathrm{b})$ wetting phase.

The reduced free energy per unit (time) length is defined as

$$
f(p, \tau ; m)=-\lim _{t \rightarrow \infty} \frac{1}{t} \ln Z_{t}(p, \tau ; m) .
$$

Fisher pointed out that an effective way to calculate $f$ is to introduce the grand generating function

$$
G(p, \tau ; m ; z)=\sum_{t=0}^{\infty} z^{t} Z_{t}(p, \tau ; m),
$$

where $z$ is the activity-like variable. Then if $z_{\min }(p, \tau ; m)$ is the positive real singularity of $G(p, \tau ; m ; z)$ nearest the origin $z=0$, then

$$
f(p, \tau ; m)=\ln z_{\min }(p, \tau ; m) .
$$

Here the singularity of $G(p, \tau, ; m ; z)$ will come from a simple pole $\sim 1 /\left(z_{\min }-z\right)$, or from a nonanalytic point, such as a square-root branch point $\sim \sqrt{z_{\min }-z}$.

For our FW system, the grand generating functions are constructed as follows. First we define sets of graphs

$$
\Gamma_{t}^{(k)}(0)=\bigcup_{\left\{x_{i}\right\}: x_{1}<x_{2}<\cdots<x_{k}} \Gamma_{t}\left(0 ; x_{1}, x_{2}, \cdots, x_{k}\right)
$$

and

$$
\Gamma_{t}(0)=\bigcup_{k: k \geq 1} \Gamma_{t}^{(k)}(0)
$$

Then (25) with (24) is rewritten as

$$
Z_{t}(p, \tau ; m)=\sum_{g: g \in \Gamma_{t}(0)} c(g ; m) \tau^{\ell(g)} p^{s(g)-1} .
$$

In general we find a site, except the origin $(0,0)$, in a graph $g \in \Gamma_{t}(0)$, such that, if we delete that site, then 
the origin $(0,0) \in V_{t}$ is disconnected from any site in $g \cap\{(x, t): x \in \mathbf{Z}\}$. Such sites are called nodal points [35].

In terms of the FW, the nodal points are the sites (except the origin at $t=0$ ) at which all $m$ walkers form a single group, (i.e., through which the trajectories of all walkers must pass). Define

$$
\begin{aligned}
& \Gamma_{t}^{N}(0 ; x)=\text { a set of all graphs in } \Gamma_{t}(0) \text { which have only one } \\
& \text { nodal point at }(x, t) \text { except at }(0,0) \\
& \Gamma_{t}^{N(1)}(0)=\bigcup_{x: x \in \mathbf{Z}} \Gamma_{t}^{N}(0 ; x), \\
& \Gamma_{t}^{N}(0)=\text { a set of all graphs in } \Gamma_{t}(0) \text { which have no nodal point } \\
& \quad \text { point except at }(0,0) .
\end{aligned}
$$

The superscript $N$ means non-nodal graphs. Then we introduce, for $m \geq 2$,

$$
G_{1}(p, \tau ; m ; z)=\sum_{t=2}^{\infty} z^{t} \sum_{g: g \in \Gamma_{t}^{N(1)}(0)} c(g ; m) \tau^{\ell(g)} p^{s(g)-1}
$$

and

$$
G_{2}(p, \tau ; m ; z)=\sum_{t=1}^{\infty} z^{t} \sum_{g: g \in \Gamma_{t}^{N}(0)} c(g ; m) \tau^{\ell(g)} p^{s(g)-1} .
$$

It should be noted that $G_{1}(p, \tau ; m ; z)$ and $G_{2}(p, \tau ; m ; z)$ are the partial generating functions for "beads" in the middle and at the end of the "necklace" of Fig.3(b). The partial generating function for "string" parts of the necklace is

$$
\begin{aligned}
G_{0}(p, \tau ; z) & =\sum_{t=0}^{\infty} z^{t}(2 p)^{t} \\
& =\frac{1}{1-2 p z}
\end{aligned}
$$

It is easy to confirm that

$$
\begin{aligned}
G(p, \tau ; m ; z) & =G_{0}+G_{0} G_{1} G_{0}+G_{0} G_{1} G_{0} G_{1} G_{0}+\cdots \\
& +G_{0} G_{2}+G_{0} G_{1} G_{0} G_{2}+G_{0} G_{1} G_{0} G_{1} G_{0} G_{2}+\cdots
\end{aligned}
$$

and for sufficiently small $z$,

$$
G(p, \tau ; m ; z)=\frac{G_{0}(p, \tau ; z)\left(1+G_{2}(p, \tau ; m ; z)\right)}{1-G_{0}(p, \tau ; z) G_{1}(p, \tau ; m ; z)} .
$$

The pole of $G(p, \tau ; m ; z)$ is determined by $1-G_{0}(p, \tau ; z) G_{1}(p, \tau ; m ; z)=0$, that is,

$$
G_{1}(p, \tau ; m ; z)=1-2 p z \text {. }
$$

Since $2 p z+G_{1}(p, \tau ; m ; z)$ is by definition increasing in $z$ (42), the equation (47) has at most one real root. We can see that the root of (47) is smaller than the pole of $G_{0}(p, \tau ; z), z_{0}=1 /(2 p)$. We will assume that the singularities of $G_{2}(p, \tau ; m ; z)$ are equal to those of $G_{1}(p, \tau ; m ; z)$. (We can see this is the case for $m=2$.) We are interested in the wetting transition as illus- trated by Fig.4. A local structure at the end of the "necklace" described by $G_{2}(p, \tau ; m ; z)$ will not affect the global structure of "necklace" at least in the nonwetting phase. Then the singularity of $G(p, \tau ; m ; z)$ may be either a simple pole at the real root of $(47)$, or the positive real singularity of $G_{1}(p, \tau ; m ; z)$. The 
problem is to determine which is smaller. If the order of these two points on the real axis of $z$ is changed as the parameters change, the change of $f(p, \tau ; m)$ may be nonanalytic, and must correspond to a phase transition.

Fisher [4] considered the following possible forms of $G_{1}(p, \tau ; m ; z)$ in the vicinity of the singularity $z_{1}=1 / w$.

$$
\begin{array}{rlr}
G_{1}(p, \tau ; m ; z) & \simeq \frac{G_{1 \mathrm{~s}}}{(1-w z)^{1-\psi}} \quad \text { for } \psi<1 \\
& \simeq G_{1 \mathrm{~s}} \ln (1-w z)^{-1} \quad \text { for } \psi=1 \\
& \simeq G_{1 \mathrm{c}}-G_{1 \mathrm{~s}}(1-w z)^{\psi-1}+G_{11}(1-w z)+\cdots \\
& & \text { for } \psi>1
\end{array}
$$

where

$$
\begin{aligned}
G_{1 \mathrm{c}} & =G_{1 \mathrm{c}}(p, \tau ; m) \\
& \equiv G_{1}(p, \tau ; m ; 1 / w)
\end{aligned}
$$

and $G_{1 \mathrm{~s}}$ is the amplitude of the leading singularity. It is concluded [4] that when $\psi \leq 1, z_{\min }=\mathrm{e}^{f}$ is always given by the real root of (47) and there is no phase transition. On the other hand, when $\psi>1$, there is a phase transition. We show in the next subsection that the latter is the case for two friendly walkers $(m=2)$, when we regard it as a model of interfacial wetting transitions.

\section{IV.2 Wetting Transitions of the $m=2$ FW}

It is easy to calculate the grand generating functions for two FW $(m=2)$ by the method of images [36, 4]. In particular, $G_{1}(p, \tau ; 2 ; z)$ is determined as

$$
\begin{aligned}
G_{1}(p, \tau ; 2 ; z) & =\left(p^{2} z\right) \times \sum_{t=1}^{\infty}\left\{\left(\begin{array}{c}
2(t-1) \\
t-1
\end{array}\right)-\left(\begin{array}{c}
2(t-1) \\
t+1
\end{array}\right)\right\}\left(p^{2} z\right)^{t-1} \times(p \tau z) \\
& =\tau p z a\left(p^{2} z\right)
\end{aligned}
$$

where $a(x)$ is the generating function of the Catalan number [37]

$$
a(x)=\frac{1}{2 x}[1-2 x-\sqrt{1-4 x}] .
$$

We can rewrite (52) as,

$$
G_{1}(p, \tau ; 2 ; z)=\frac{\tau}{4 p}-\frac{\tau}{2 p}\left(1-4 p^{2} z\right)^{1 / 2}+\frac{\tau}{4 p}\left(1-4 p^{2} z\right) .
$$

Then the third case (50) is realized with

$$
\frac{1}{z_{1}}=w=(2 p)^{2} \quad \text { and } \quad \psi=\frac{3}{2} .
$$

It should be noted that a square-root branch-point $z_{1}=1 / w=1 /(2 p)^{2}$ is a singular point but $G_{1}(p, \tau ; 2 ; z)$ remains finite as $z \rightarrow z_{1}-: G_{1 \mathrm{c}}(p, \tau ; 2)=\tau /(4 p)<\infty$.

Now we determine the wetting transition point of two FW $(m=2)$ following the theory of Fisher. First we consider the special case in which $\tau=1$. Fig. 5 (a) shows $y=G_{1}(p, 1 ; 2 ; z)$ and $y=1-2 p z$ as functions of $z$ for $p=0.6$. The crossing point gives a root of the equation (47), which is smaller than $z_{1}=1 /(2 \times 0.6)^{2}=$ $0.694 \cdots$ On the other hand, when $p=0.8,(47)$ has no real root, as shown in Fig.5 (c). Figure 5 (b) shows the critical case with $p=p_{\mathrm{cw}}=3 / 4$. In this case, a line $y=1-2 p z$ goes through a critical point $\left(z_{1}, G_{1 \mathrm{c}}(p, 1 ; 2)\right)=\left(1 /\left(2 p_{\mathrm{cw}}\right)^{2}, 1 /\left(4 p_{\mathrm{cw}}\right)\right)=(4 / 9,1 / 3)$. 
Then for the case $\tau=1$, when $p<3 / 4$, the reduced free energy $f$ is given by the root of (47) and the system is non-wetting (the case of Fig.4 (a)), and when $p>3 / 4, f=\ln z_{1}=-2 \ln (2 p)$ and the system is wetting (the case of Fig.4(b)). The wetting transition occurs at $p=3 / 4$. In the general case $\tau \geq 0$, we can conclude the following.

$$
\begin{aligned}
& \frac{\tau}{4 p}<1-\frac{1}{2 p} \Longleftrightarrow \text { wetting } \\
& \frac{\tau}{4 p}>1-\frac{1}{2 p} \Longleftrightarrow \text { non-wetting. }
\end{aligned}
$$

For each $\tau \geq 0$, the critical value of $p$ at which the wetting transition occurs is given by

$$
p_{\mathrm{cw}}(\tau)=\frac{\tau}{4}+\frac{1}{2}
$$

For $p<p_{\mathrm{cw}}$ the real root of $(47)$, at which $G(p, \tau ; 2 ; z)$ has a simple pole, is given by $z_{\text {pole }}(p, \tau)=\frac{1}{(2-\tau)^{2} p^{2}}[(2-\tau) p-\tau+\tau \sqrt{(\tau-2) p+1}]$

Since

$$
z_{\text {pole }}(p, \tau)-\frac{1}{(2 p)^{2}} \simeq-\frac{16}{(\tau+2)^{2} \tau^{2}}\left(p_{\text {cw }}(\tau)-p\right)^{2}
$$

for $0<p_{\mathrm{cw}}(\tau)-p \ll 1$, we have the reduced free energy in the form

$$
f(p, \tau ; 2)=f_{\mathrm{r}}(p, 2)+f_{\mathrm{s}}(p, \tau ; 2)
$$

with a regular part

$$
f_{\mathrm{r}}(p, 2)=-2 \ln (2 p)
$$

and a singular part

$$
f_{\mathrm{s}}(p, \tau ; 2)= \begin{cases}-\frac{4}{\tau^{2}}\left(p_{\mathrm{cw}}(\tau)-p\right)^{2}+\cdots & \text { for } p \leq p_{\mathrm{cw}}(\tau) \\ 0 & \text { for } p>p_{\mathrm{cw}}(\tau)\end{cases}
$$
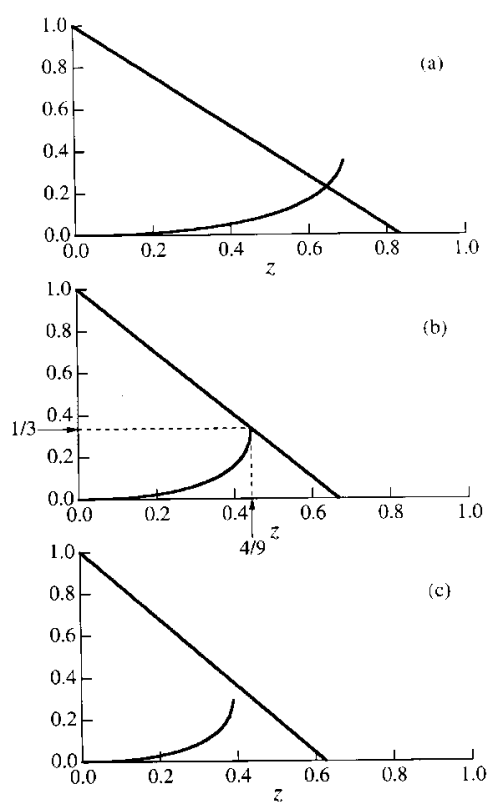

Figure 5. The curves $y=G_{1}(p, 1 ; 2 ; z)$ and lines $y=1-2 p z$ for (a) $p=0.6$, (b) $p=3 / 4$ and (c) $p=0.8$.

If we define the specific-heat critical exponent $\alpha$ through $f_{\mathrm{s}} \sim\left(p_{\mathrm{cw}}(\tau)-p\right)^{2-\alpha}$, we have

$$
\alpha=0 \text {. }
$$

Fisher gave a general scaling relation

$$
\alpha=\frac{2 \psi-3}{\psi-1}
$$

where $\psi$ is the exponent in (48)-(50) [4].

The result (30) shows that if we set $p=p_{1}$ and $\tau=\left(2 p_{1}-p_{2}\right) / p_{1}$, and take the $m \rightarrow 0$ limit, then FW becomes the Domany-Kinzel model. Here we set $p=p_{1}, \tau=\left(2 p_{1}-p_{2}\right) / p_{1}$ for the two FW $(m=2)$. Fig. 6 shows the phase diagram of two $\mathrm{FW}$ in the $\left(p_{1}, p_{2}\right)$-plane. The critical line $(57)$ is now given by $p_{1}=p_{1 \mathrm{cw}}\left(p_{2}\right)$ with

$$
p_{1 \mathrm{cw}}\left(p_{2}\right)=\frac{1}{2}+\frac{1}{2} \sqrt{1-p_{2}} \text {. }
$$

It should be noted that $p_{1}=p_{1 \mathrm{cw}}\left(p_{2}\right)$ coincides with the line $p_{1}=p_{1 \mathrm{u}}\left(p_{2}\right)$ given by (18) with (17), which gives the upper bound for the DP critical line of the Domany-Kinzel model in the region $p_{1} \leq p_{2} \leq 2 p_{1}$ [26]. 


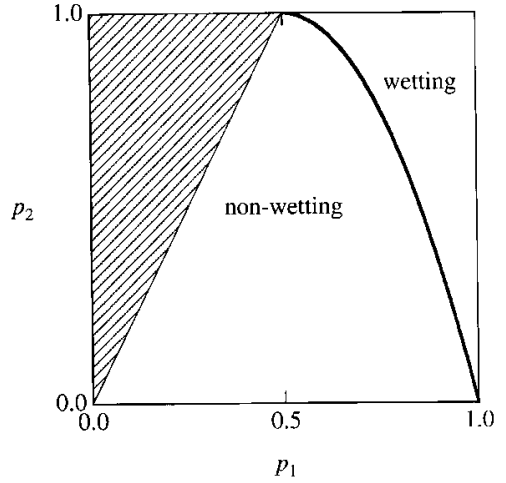

Figure 6 . The phase diagram of the two FW $(m=2)$ on the $\left(p_{1}, p_{2}\right)$-phase diagram. The hatched region, $p_{2}>2 p_{1}$, should be ignored, since $\tau<0$ there.

To close this section, we give an interpretation of the results for two $\mathrm{FW}$ again from the viewpoint of the wetting transition. When $m=2$, the system is a twodimensional, three-phase system composed of $A, B$ and
$C$ phases (see Fig.4). If we write the surface tensions of the $A \mid B$ and $B \mid C$ interfaces at temperature $T$ as $\Sigma_{A B}(T)$ and $\Sigma_{B C}(T)$, respectively, the regular part of the reduced free energy may be written as

$$
f_{\mathrm{r}}(p ; 2)=\left(\Sigma_{A B}(T)+\Sigma_{B C}(T)\right) / k_{\mathrm{B}} T .
$$

Comparing this with (61), we can put $\Sigma_{A B}(T)=$ $\Sigma_{B C}(T)=-k_{B} T \ln (2 p)$ or

$$
2 p=\mathrm{e}^{-\Sigma_{A B}(T) / k_{\mathrm{B}} T}=\mathrm{e}^{-\Sigma_{B C}(T) / k_{\mathrm{B}} T} .
$$

Therefore, changing the parameter $p$ corresponds to changing the temperature $T$ of the system. On the other hand, the parameter $\tau$ can be regarded as an activity associated with the end of the "beads" of $B$ phase. Let $T_{\mathrm{cw}}(\tau)$ be the critical temperature of wetting transition corresponding to $p_{\mathrm{cw}}(\tau)$ and $\Sigma_{A C}(T)$ be the surface tension between the bulk $\mathrm{A}$ and $\mathrm{C}$ phases. Then (60)-(62) gives

$$
\begin{aligned}
& \Sigma_{A C}(T) \\
& \quad= \begin{cases}\Sigma_{A B}(T)+\Sigma_{B C}(T)-A_{0}\left(T_{\mathrm{cw}}(\tau)-T\right)^{2}+\cdots & \text { for } T \leq T_{\mathrm{cw}}(\tau) \\
\Sigma_{A B}(T)+\Sigma_{B C}(T) & \text { for } T>T_{\mathrm{cw}}(\tau)\end{cases}
\end{aligned}
$$

with a constant $A_{0}$. This description of interfacial wetting transitions in two-dimensional fluids is found in $[4]$.

\section{FW as an Interacting Vicious Walkers and Vertex Model}

\section{V.1 Interacting Vicious Walkers}

In this section, we consider the $m \mathrm{FW}$ with a condition

$$
\tau=p
$$

which corresponds to DBP when we put $p=p_{1}$, $\tau=\left(2 p_{1}-p_{2}\right) / p_{1}$ and $m=0$. Using Euler's law $\ell(g)=b(g)-s(g)+1,(38)$ becomes

$$
Z_{t}(p, p ; m)=\sum_{g: g \in \Gamma_{t}(0)} c(g ; m) p^{b(g)} .
$$

As shown in Fig. 7(a), we consider $m$ FW, in which all walkers start from the origin and the non-crossing condition (21) has been imposed. For each set of trajectories of $m \mathrm{FW}$, we can make a set of trajectories by shifting the $i$-th trajectory to the right by $(i-1)$ lattice-units as shown in Fig. 7(b). Such a graph is called a polymer brush with $m$ branches [6].

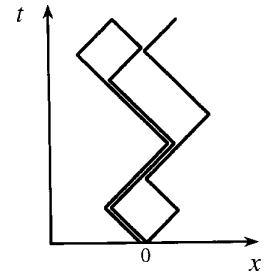

(a)

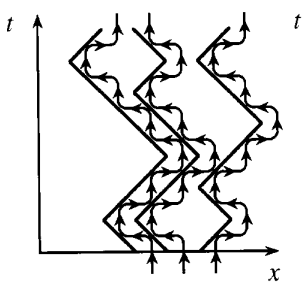

(c)

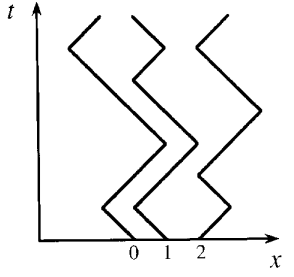

(b)

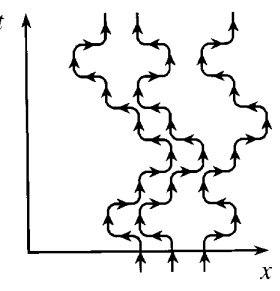

(d)
Figure 7. Construction of the vertex model from the $m$ FW.

The non-crossing condition is mapped to the condition with strict inequalities,

$$
x_{1}(s)<x_{2}(s)<\cdots<x_{m}(s) .
$$


Walkers subject to the condition (71) are described as vicious walkers $[4,5,6,38,39]$, since they can be regarded as the surviving walkers who shoot each other dead (i.e., pair annihilation) when whey arrive at the same site $\left(x_{i}(s)=x_{i+1}(s)\right.$ for some $\left.i\right)$. If $p=1,(70)$ is the total number of ways of $m$ vicious walkers to walk such that they all survive for at least $t$ time-steps starting from the sites $x_{i}=i-1(i=1, \cdots, m)$.

Let $h(g)$ be the number of pairs of bonds which run parallel to each other, maintaining a distance of one lattice unit $(h(g)=6$ for Fig. $7(\mathrm{~b}))$. Then $p^{b(g)}=p^{m t} \times p^{-h(g)}$ for $m$ walkers up to time $t$. It means that the $m \mathrm{FW}$ model is an interacting vicious walker model, in which if a pair of nearest-neighboring walkers walk parallel to each other for one time step, the walk is weighted by $p^{-1}$. (If three neighbors walk parallel to one another, they are regarded as two pairs and the weight carries a factor of $p^{-2}$.) Since $p^{-1}>1$, the walkers prefer to walk parallel to one another. Thus we can say that these vicious walkers are friendly to their neighbors, as long as they don't get too close!

\section{V.2 Ten-Vertex Model}

Interacting vicious walkers can be described by a kind of vertex model. For each trajectory of the interacting vicious walkers, draw a right contour as shown in Fig. 7(c). Then erase the trajectories of the interacting vicious walkers and identify a set of contours as a line of configurations of a vertex model (Fig. 7(d)). Note that all lines have directions which are represented by arrows at each bond (segments of lines).

We introduce a ten-vertex model. The ten local $d i$ rected line configurations at a vertex are shown in Fig. 8. Each bond is in one of the four states represented by an upward arrow, a leftward arrow, a rightward arrow, or nothing. Down arrows are forbidden and the noncrossing condition is imposed. We assign the weights $a_{0}, a_{\mathrm{r}}, a_{\ell}, b_{0}, b_{\mathrm{r}}, b_{\ell}, c_{\mathrm{r}}, c_{\ell}, c_{\mathrm{r}}^{\prime}, c_{\ell}^{\prime}$ for the ten configurations, as shown in Fig.8. We assume the initial configuration (the boundary condition at the bottom on a spatiotemporal plane $V_{t}$ ), such that there are $m$ upward lines at $x=0,1,2, \cdots, m-1$, and sum up all configurations of directed lines with appropriate weights in which $m$ directed lines are conserved up to time $t$. There is no restriction on the configurations at the final time $t$. The partition function of the ten-vertex model is defined as

$$
Z_{t}^{10 v}\left(a_{0}, a_{\mathrm{r}}, a_{\ell}, b_{0}, b_{\mathrm{r}}, b_{\ell}, c_{\mathrm{r}}, c_{\ell}, c_{\mathrm{r}}^{\prime}, c_{\ell}^{\prime} ; m\right)=\sum_{\text {allowed config. }} \prod(\text { weights }) .
$$

The construction of the vertex model for $m \mathrm{FW}$ shown in Fig.7 yields the identity

$$
Z_{t}(p, p ; m)=p^{m t} Z_{t}^{10 \mathrm{v}}\left(1, \frac{1}{p}, \frac{1}{p}, 0,0,0,1,1,1,1 ; m\right) .
$$

Since all straight lines across a vertex are forbidden, $b_{0}=b_{\mathrm{r}}=b_{\ell}=0$, and the left-right symmetry relations, $a_{\mathrm{r}}=a_{\ell}(=1 / p), c_{\mathrm{r}}=c_{\ell}(=1)$, and $c_{\mathrm{r}}^{\prime}=c_{\ell}^{\prime}(=1)$, are satisfied, we can say that the $m \mathrm{FW}$ with (69) can be mapped to a symmetric seven-vertex model.

If we consider the totally asymmetric case such that $a_{\ell}=b_{\ell}=c_{\ell}=c_{\ell}^{\prime}=0$, where all leftward lines are forbidden, the arrows put on bonds can be erased and the configurations are represented by undirected lines. In this case the system is identified with the six-vertex model [40],

$$
Z_{t}^{10 \mathrm{v}}(a, a, 0, b, b, 0, c, 0, c, 0 ; m)=Z_{t}^{6 \mathrm{v}}(a, b, c ; m) .
$$

As shown in [40], the six-vertex model can be mapped to the critical $q$-state Potts model. We have the famous result by Kasteleyn and Fortuin [41, 42], that for an arbitrary undirected finite lattice, the percolation problem can be formulated using the $q \rightarrow 1$ limit of the $q$-state Potts models. Then we can conclude that the undirected percolation problem can be formulated in terms of the totally asymmetric (six-)vertex model.

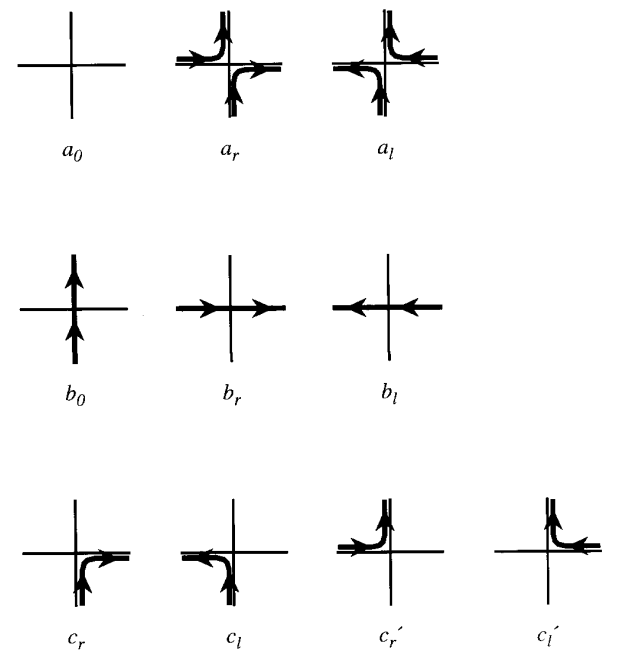

Figure 8. The ten kinds of directed-line configurations in the ten-vertex model with their weights.

On the other hand, we have shown that $m \mathrm{FW}$ with 
(69), whose $m=0$ limit gives the DBP system, is mapped to the symmetric (seven-) vertex model. The following correspondence is now clarified.

$$
\begin{aligned}
& \text { Percolation Vertex Model } \\
& \text { undirected } \Longleftrightarrow \text { asymmetric } \\
& \text { directed } \Longleftrightarrow \text { symmetric }
\end{aligned}
$$

It is interesting to see that directed percolation, which can be defined as a process with time-irreversibility, (that is, totally asymmetric in time direction) corresponds to the symmetric case of the vertex model.

\section{V.3 Bethe Ansatz and Exact Solvability}

The six-vertex model can be solved exactly by the Bethe ansatz [40]. We can see that the total asymmetry of the six-vertex model is essential to allow us to use the Bethe ansatz to solve the problem. We will show here, however, an attempt to apply the Bethe ansatz method to the symmetric vertex model. First we consider a finite square lattice with $t$ rows ( $t$ steps in the time direction) and $L$ columns ( $L$ sites in the spatial direction) and assume periodic boundary conditions in the spatial direction. The state $X$ on each row is specified by the locations of $m$ lines on the $L$ sites, $X=\left\{x_{1}, x_{2}, \cdots, x_{m}\right\}$. Let $\mathcal{T}$ be the row-to-row transfer matrix of our ten-vertex model, $\Lambda$ an eigenvalue of $\mathcal{T}$, and $g$ the corresponding eigenfunction, then

$$
\Lambda g(X)=\sum_{Y} \mathcal{T}(X, Y) g(Y)
$$

When $t$ is large, $Z_{t}^{10 \mathrm{v}} \sim \Lambda_{\text {max }}^{t}$, where $\Lambda_{\max }$ is the maximum eigenvalue of $\mathcal{T}$.

The $m=1$ case is trivial. For $m=2$, we assume the form of the Bethe ansatz for $g(X)$,

$$
g\left(x_{1}, x_{2}\right)=A_{12} \xi_{1}^{x_{1}} \xi_{2}^{x_{2}}+A_{21} \xi_{1}^{x_{2}} \xi_{2}^{x_{1}},
$$

where $A_{12}, A_{21}, \xi_{1}, \xi_{2}$ are complex numbers. We find for the symmetric seven-vertex model, $\left(a_{0}, a_{\mathrm{r}}, a_{\ell}, b_{0}, b_{\mathrm{r}}, b_{\ell}, c_{\mathrm{r}}, c_{\ell}, c_{\mathrm{r}}^{\prime}, c_{\ell}^{\prime}\right)=(1,1 / p, 1 / p, 0,0,0$, $1,1,1,1)$, that if the Yang-Baxter relations

$$
\begin{aligned}
& \xi_{1}^{N}=\frac{A_{12}}{A_{21}}, \quad \xi_{2}^{N}=\frac{A_{21}}{A_{12}}, \\
& \frac{A_{12}}{A_{21}}=-\frac{\xi_{2}\left[(1-p)\left(\xi_{1}^{2}+1 / \xi_{2}^{2}\right)-p\right]}{\xi_{1}\left[(1-p)\left(\xi_{2}^{2}+1 / \xi_{1}^{2}\right)-p\right]}
\end{aligned}
$$

are satisfied, then

$$
\Lambda\left(\xi_{1}, \xi_{2}\right)=\left(\xi_{1}+\frac{1}{\xi_{1}}\right)\left(\xi_{2}+\frac{1}{\xi_{2}}\right) .
$$

The maximum eigenvalue $\Lambda_{\max }$ will be obtained by setting $\xi_{1} \xi_{2}=1$ and the solution of (78) determines, in the $L \rightarrow \infty$ limit,

$$
\Lambda_{\max }(p)=\frac{(p-2)^{2}}{2 p(1-p)} .
$$

Then (73) gives

$$
Z_{t}(p, p ; 2) \sim \lambda_{\max }^{t} \quad \text { for } \quad t \gg 1
$$

with

$$
\begin{aligned}
\lambda_{\max }(p) & =p^{2} \Lambda_{\max }(p) \\
& =\frac{p(p-2)^{2}}{2(1-p)}
\end{aligned}
$$

It should be noted that the relation

$$
\lambda_{\max }(p)=\frac{1}{z_{\text {pole }}(p, p)}
$$

holds, where $z_{\min }(p, p)$ is given by (58) with $\tau=p$. That is, in the case of $m=2$ with (69), the Bethe ansatz method determines the location of the pole of $G(p, \tau ; 2 ; z)$.

As shown in Section IV, to discuss wetting transitions we have to know not only the location of the pole but also the singular point of $G_{1}(p, \tau ; 2 ; z)$. When $m=2$, the trajectories of the two FW which contribute to $G_{1}(p, \tau ; 2 ; z)$ are represented by lines of the ten-vertex model with $\left(a_{0}, a_{\mathrm{r}}, a_{\ell}, b_{0}, b_{\mathrm{r}}, b_{\ell}, c_{\mathrm{r}}, c_{\ell}, c_{\mathrm{r}}^{\prime}, c_{\ell}^{\prime}\right)$ $=(1,0,0,0,0,0,1,1,1,1)$. If two lines run separately, they seem to be the trajectories of two independent random walkers, who step either to the right or left at random, but if two lines meet at a vertex, they are pair-annihilated. We expect that $Z_{t}^{10 \mathrm{v}}(1,0,0,0,0,0,1,1,1,1 ; 2) \sim 2^{2 t} / t^{\psi}$ with an exponent $\psi$ (the number of trajectories $2^{2 t}$ is reduced by a factor $1 / t^{\psi}$ due to pair-annihilation) [4]. It follows that $G_{1}(p, \tau ; 2 ; z)=\sum_{t} z^{t} p^{2 t} Z_{t}^{10 \mathrm{v}} \sim \sum_{t}\left((2 p)^{2} z\right)^{t} / t^{\psi} \sim$ $\left(1-(2 p)^{2} z\right)^{\psi-1}$. Then the singular point $G_{1}(p, \tau ; 2 ; z)$ can be determined as $z_{1}(p)=1 /(2 p)^{2}$. Using the result (82) of the Bethe ansatz, we have

$$
\begin{aligned}
\frac{1}{\lambda_{\max }(p)}-z_{1}(p) & =-\frac{9}{4 p^{2}(p-2)^{2}}\left(\frac{2}{3}-p\right)^{2} \\
& \simeq-\frac{3^{6}}{2^{8}}\left(\frac{2}{3}-p\right)^{2}
\end{aligned}
$$

for $0<2 / 3-p \ll 1$. If we put $\tau=p$ in (57) and (59), we obtain the same result as (84) from them. This means that (84) determines $p_{\mathrm{cw}}=2 / 3$ and $\psi=2 / 3$ (i.e. $\alpha=0)$ in the case (69).

In the present paper we have shown two ways to solve the two FW model $(m=2)$ : by a combinatorial method in Section IV.B and by the Bethe ansatz via mapping to a vertex model here. As far as we know, 
there are no exact results for $m \geq 3$. As far as we know, there are no exact results for $m \geq 3$. As we can imagine when we look at Fig.4, the $B_{i}$ 's phases will have complex structures inside them if $m$ is large, and we expect a rich physics of wetting transitions in FW models with $m \geq 3$.

\section{Acknowledgments}

The author would like to thank Norio Inui for useful discussions on the friendly walker problem and wetting transitions. A part of this work was supported by a Grant-in-Aid for Scientific Research from the Ministry of Education, Science and Culture, Japan.

\section{References}

[1] J. Marro and R. Dickman, Nonequilibrium Phase Transitions in Lattice Models, (Cambridge University Press, Cambridge, 1999).

[2] E. Domany and W. Kinzel, Phys. Rev. Lett. 53, 311 (1984).

[3] T. Tsuchiya and M. Katori, J. Phys. Soc. Jpn. 67, 1655 (1998).

[4] M. E. Fisher, J. Stat. Phys. 34, 667 (1984).

[5] D. K. Arrowsmith, P. Mason and J. W. Essam, Physica A 177, 267 (1991).

[6] J. W. Essam and A. J. Guttmann, Phys. Rev. E 52, 5849 (1995).

[7] W. Kinzel, Z. Phys. B 58, 229 (1985).

[8] B. Chopard and M. Droz, Cellular Automata Modeling of Physical Systems, (Cambridge University Press, Cambridge, 1998).

[9] T. M. Liggett, Interacting Particle Systems (Springer, New York, 1985).

[10] R. Durrett, Lecture Notes on Particle Systems and Percolation (Wadsworth and Brooks/Cole, Pacific Grove, CA, 1988) Section 5b.

[11] N. Konno, Phase Transitions of Interacting Particle Systems (World Scientific, Singapore, 1994).

[12] T. M. Liggett, Stochastic Interacting Systems: Contact, Voter and Exclusion Processes, (Springer, New York, 1999).

[13] R. Durrett and D. Griffeath, Ann. Probab. 11, 1 (1983).

[14] M. Katori and N. Konno, J. Phys. A: Math. Gen. 26, 6597 (1993).

[15] I. Jensen and R. Dickman, Physica A 203, 175 (1994).

[16] T. E. Harris, Ann. Probab. 2, 969 (1974).

[17] T. M. Liggett, The periodic threshold contact process, in Random Walks, Brownian Motion and Interacting Particle Systems, edited R. Durrett and H. Kesten (Birkhäuser, Boston, 1991) pp. 339-358.
[18] R. C. Brower, M. A. Furman and M. Moshe, Phys. Lett. B 76, 213 (1978).

[19] P. Grassberger and A. de la Torre, Ann. Phys. 122, 373 (1979).

[20] J. L. Cardy and R. L. Sugar, J. Phys. A: Math. Gen. 13, L423 (1980).

[21] R. Dickman and M. A. Burschka, Phys. Lett. A 127, 132 (1988).

[22] R. M. Ziff, E. Gulari and Y. Barshad, Phys. Rev. Lett. 56, 2553 (1986).

[23] R. Dickman and T. Tomé, Phys. Rev. A 44, 4833 (1991).

[24] M. Katori, J. Phys. A: Math. Gen. 27, 3191 (1994).

[25] M. Katori and H. Tsukahara, J. Phys. A: Math. Gen. 28, 3935 (1995).

[26] T. M. Liggett, Ann. Appl. Probab. 5, 613 (1995).

[27] D. Dhar, J. Phys. A: Math. Gen. 15, 1849 (1982).

[28] I. Jensen and A. J. Guttmann, J. Phys. A: Math. Gen. 28, 4813 (1995).

[29] J. Cardy and F. Colaiori, Phys. Rev. Lett. 82, 2232 (1999).

[30] M. Katori, T. Tsuchiya, N. Inui and H. Kakuno, Annals of Combinatorics 3, 337 (1999).

[31] D. K. Arrowsmith and J. W. Essam, J. Math. Phys. 18, 235 (1977).

[32] J. W. Essam, Percolation and cluster size, in Phase Transitions and Critical Phenomena, vol.2, C. Domb and M. S. Green, eds., (Academic Press, London, 1972) pp.197-270.

[33] J. W. Essam, Rep. Prog. Phys. 43, 833 (1980).

[34] N. Konno, in preparation.

[35] F. M. Bhatti and J. W. Essam, J. Phys. A: Math. Gen. 17, L67 (1984).

[36] D. A. Huse, A. M. Szpilka and M. E. Fisher, Physica A 121, 363 (1983).

[37] N. Inui and M. Katori, J. Phys. A: Math. Gen. 29, 4347 (1996).

[38] A. J. Guttmann, A. L. Owczarek and X. G. Viennot, J. Phys. A: Math. Gen. 31, 8123 (1998).

[39] A. J. Guttmann and M. Vöge, Lattice paths: Vicious walkers and friendly walkers, submitted to J. Stat. Inference and Planning (1999).

[40] R. J. Baxter, Exactly Solved Models in Statistical Mechanics (Academic Press, London, 1982).

[41] P. W. Kasteleyn and C. M. Fortuin, J. Phys. Soc. Jpn. Suppl. 26, 11 (1969).

[42] C. M. Fortuin and P. W. Kasteleyn, Physica 57, 536 (1972). 